EPJ manuscript No.

(will be inserted by the editor)

\title{
Interactions Between Charged Rods Near Salty Surfaces
}

\author{
Rebecca Menes ${ }^{1}$ Niels Grønbech-Jensen ${ }^{2}$ and Phil A. Pincus ${ }^{1}$ \\ 1 Materials Research Laboratory, University of California, Santa Barbara, California, 93106, USA \\ 2 Department of Applied Science, University of California, Davis, California 95616, USA \\ NERSC, Lawrence Berkeley National Laboratory, Berkeley, California 94720, USA
}

Received: date / Revised version: date

\begin{abstract}
Using both theoretical modeling and computer simulations we study a model system for DNA interactions in the vicinity of charged membranes. We focus on the polarization of the mobile charges in the membranes due to the nearby charged rods (DNA) and the resulting screening of their fields and inter-rod interactions. We find, both within a Debye-Hückel model and in Brownian dynamics simulations, that the confinement of the mobile charges to the surface leads to a qualitative reduction in their ability to screen the charged rods to the degree that the fields and resulting interactions are not finite-ranged as in systems including a bulk salt concentration, but rather decay algebraically and the screening effect is more like an effective increase in the multipole moment of the charged rod.
\end{abstract}

PACS. PACS-key describing text of that key - PACS-key describing text of that key

\section{Introduction}

Recent experiments on various systems containing DNA strands and charged surfaces have raised an interest in understanding how these surfaces screen the electrostatic fields of the DNA and the resulting interactions between strands. The interest emanates from sources varying from understanding prokaryotic DNA replication [1], to non-viral gene therapy [2, $3,4,5$, and even DNA chip technology[6.

Send offprint requests to:
In this paper we treat this problem using a two-dimensional salt solution model[7, 8, 9, [0, 11] to account for the charged surface (membrane), while the DNA strands are modeled as negatively charged rigid rods. We treat charge neutral systems where the over all charging of the surface and the rod is zero, thus focusing either on overall neutrally charged mixed lipid fluid membranes 12, or highly charged surfaces to which the counter-ions are strongly bound and therefore treated within a two-dimensional ge- 
ometry. We neglect the possible dependence on dielectric properties of the components in the experimental systems mentioned above in order to simplify the theoretical picture. The effects of the thickness and the dielectric properties of the layers will be published elsewhere 13 .

We focus on very simple geometries in order to understand how the DNA strand and surface charges interact and screen. The geometry is that of one, infinite, salty surface decorated with either one or two DNA strands. We calculate the charge distribution around the DNA and the resulting interaction between the two strands. We calculate the interaction assuming the DNA strands are slightly raised above the surface (See Fig. 1).

The theory we use to obtain our analytical results is within the Debye-Hückel approximation 14: We minimize the free energy of the system with respect to the charge densities and use the result in the Poisson equation (thus obtaining the Poisson-Boltzmann (PB) equation) which we linearize with respect to the electrostatic potential. Solving this equation leads to the optimized self consistent charge distribution which we can insert back into the free energy in order to obtain the resulting interactions. In the next section we introduce the model and the formal results, and in the following section we apply it to find the interactions between two strands. We compare these results with new simulations of two-dimensional salt solutions and we conclude with a discussion on the limits of applicability and relevance of this model.

\section{Model}

The free energy of a system of fixed and mobile charges includes electrostatic terms and entropic terms:

$F_{\text {elec }}=\frac{1}{2} \frac{e^{2}}{\epsilon} \int\left(\frac{\sigma(\mathbf{r}) \sigma\left(\mathbf{r}^{\prime}\right)}{\left|\mathbf{r}-\mathbf{r}^{\prime}\right|}+\frac{\Sigma(\mathbf{r}) \Sigma\left(\mathbf{r}^{\prime}\right)}{\left|\mathbf{r}-\mathbf{r}^{\prime}\right|}+2 \frac{\sigma(\mathbf{r}) \Sigma\left(\mathbf{r}^{\prime}\right)}{\left|\mathbf{r}-\mathbf{r}^{\prime}\right|}\right) d \mathbf{r} d \mathbf{r}^{\prime}$

$S=-\int\left(\sigma_{+}(\mathbf{r})\left(\log \left(\sigma_{+}(\mathbf{r}) a_{0}\right)-1\right)+\sigma_{-}(\mathbf{r})\left(\log \left(\sigma_{-}(\mathbf{r}) a_{0}\right)-1\right)\right) d \mathbf{r}$

Here $\sigma_{+}$and $\sigma_{-}$are the number densities of the positive and negative mobile charges where the total mobile charge density is given by $e \sigma=e \sigma_{+}-e \sigma_{-}$, and $e \Sigma=e \Sigma_{+}-e \Sigma_{-}$are the fixed charge densities.

Minimizing the Grand Potential:

$$
G=F_{\text {elec }}-k_{B} T S-\int \mu\left(\sigma_{+}+\sigma_{-}\right) d \mathbf{r}
$$

with respect to the mobile charge densities, $\sigma_{+}$and $\sigma_{-}$, yields:

$$
\sigma_{+}(\mathbf{r})=a_{0}^{-1} e^{\frac{-e \phi(\mathbf{r})+\mu}{k_{B} T}} \quad \sigma_{-}(\mathbf{r})=a_{0}^{-1} e^{\frac{e \phi(\mathbf{r})+\mu}{k_{B} T}}
$$

Here the chemical potential of the positive and negative charges, $\mu$, is taken to be equal since we treat the thermodynamic limit of an infinite system where the average number of positive and negative charges are equal far from the rods, and

$$
\phi(\mathbf{r})=\frac{e}{\epsilon} \int\left(\frac{\sigma_{+}\left(\mathbf{r}^{\prime}\right)-\sigma_{-}\left(\mathbf{r}^{\prime}\right)+\Sigma_{+}\left(\mathbf{r}^{\prime}\right)-\Sigma_{-}\left(\mathbf{r}^{\prime}\right)}{\left|\mathbf{r}-\mathbf{r}^{\prime}\right|}\right) d \mathbf{r}^{\prime}
$$

is the resulting electrostatic potential. Inserting these results into the free energy we find the formal expression:

$$
G=\frac{1}{2} \int(\Sigma(\mathbf{r})-\sigma(\mathbf{r})) \phi(\mathbf{r}) d \mathbf{r}
$$


In this expression we have dropped constant terms that do not depend on the exact geometry of the system since we are interested in how the energy depends on the distances between the charges objects. We will use this expression in the next section to calculate the interactions in this system. Note that the mobile charge density, $\sigma$, enters with an opposite sign to what one would have naively guessed to be the interaction. This is due to the fact that this term enters as an entropic contribution and therefore indicates how the entropy has been reduced (and thus the free energy increased) due to the arrangement of the mobile charges around the fixed charges.

By inserting the distributions of Eq. 1 in the Poisson equation we get the Poisson Boltzmann equation 15]:

$$
\epsilon \nabla^{2} \phi(\mathbf{r})=-4 e \pi\left(\left(e^{\frac{-e \phi(\mathbf{r})+\mu}{k_{B} T}}-e^{\frac{e \phi(\mathbf{r})+\mu}{k_{B} T}}\right) a_{0}^{-1}+\Sigma(\mathbf{r})\right) \delta(z)
$$

The $\delta$ function was introduced because the charges are confined to the surface at $z=0$. Linearizing this equation yields the Debye-Hückel (DH) equation 15]:

$$
\epsilon \nabla^{2} \phi(\mathbf{r})=\left(\frac{1}{\lambda} \phi(\mathbf{r})-4 \pi e \Sigma(\mathbf{r})\right) \delta(z) \quad ; \quad \frac{1}{\lambda}=\frac{8 e^{2} \pi e^{\frac{\mu}{k_{B} T}}}{k_{B} T a_{0}}
$$

Solving Eq. 8 for a given fixed charge distribution $\Sigma(\mathbf{r})$, yields the mobile charge distribution and electrostatic potentials and fields. In our case the fixed charge is that of a uniformly charged stiff rod (model DNA). We first solve Eq. 8 for a fixed point charge $Q$, (i.e., $\Sigma(\mathbf{r})=Q \delta(\rho) \delta(z))$ and then, since the problem is linear, we integrate to find the corresponding solution for an infinitely long rod.
The potential that solves Eq. 8 has two contributions: a singular part, $\phi_{\text {sing }}=\frac{Q}{\epsilon \sqrt{\rho^{2}+z^{2}}}$, which solves the equation for a single point charge: $\epsilon \nabla^{2} \phi_{\text {sing }}(\mathbf{r})=-4 e \pi Q \delta(\rho) \delta(z)$. The second contribution, $\psi$, arises from the mobile charges on the surface and must satisfy the remaining equation:

$$
\epsilon \nabla^{2} \psi(\mathbf{r})=\frac{1}{\lambda}\left(\phi_{\text {sing }}+\psi\right) \delta(z) .
$$

Eq. 9 is the Laplace equation: $\nabla^{2} \psi=0$, with the special boundary condition at $z=0$ :

$$
\frac{\partial \epsilon \psi\left(0_{+}\right)}{\partial z}-\frac{\partial \epsilon \psi\left(0_{-}\right)}{\partial z}=\left.\frac{e}{\lambda}\left(\psi+\phi_{s i n g}\right)\right|_{z=0} .
$$

We solve for $\psi$ with a family of solutions of the Laplace equation:

$$
\psi(\mathbf{r})=\int \alpha_{q} e^{-q|z|} J_{0}(q \rho) d q .
$$

Using the identity $\phi_{\text {sing }}=\frac{Q}{\epsilon \sqrt{\rho^{2}+z^{2}}}=\frac{Q}{\epsilon} \int e^{-q|z|} J_{0}(q \rho) d q$, the boundary condition (Eq. 10) is easily satisfied with $\alpha_{q}=-\frac{Q / \epsilon}{2 q \epsilon \lambda+1}$, and the total electrostatic potential of the system is given by:

$$
\phi_{\text {point }}=\phi_{\text {sing }}+\psi=Q \int e^{-q|z|} J_{0}(q \rho) \frac{2 q \lambda}{2 \epsilon q \lambda+1} d q .
$$

This potential can now be integrated over a line to give the potential of a charged rod on a salty surface:

$$
\phi_{\text {rod }}=\int \phi_{\text {point }}(\mathbf{r}) d y=2 \tau \int e^{-q|z|} \frac{\cos (q x)}{q} \frac{2 q \lambda}{2 \epsilon q \lambda+1} d q
$$

where $\tau$ is the charge per unit length on the bare rod (in the case of DNA: $\tau \simeq-e / 1.7 \AA)$. Within this model the resulting charge distribution on the surface is found to be:

$$
\sigma(x)=-\frac{1}{2 \pi \lambda} \phi_{\text {rod }}=-\frac{\tau}{\pi} \int \frac{\cos (q x) d q}{2 \epsilon q \lambda+1} .
$$




\section{Interactions}

In this section we calculate the effective interactions between the components in the system. The surface charges, which distribute themselves around the fixed charges, screen to some extent their fields and thus the direct interactions. However, the screening is not as effective as that of a threedimensional salt solution where the exponential screening leads to a finite ranged interaction. In the case of a twodimensional salt, although reduced, the fields are still long ranged[7]. This can be seen when we take the limit of large distances from the rod and calculate the fields resulting from Eq. 13]10]:

$$
E_{x}(x \gg \lambda, z=0) \simeq \frac{8 \epsilon^{2} \tau \lambda^{2}}{x^{3}} \quad E_{z}(x=0, z \gg \lambda) \simeq \frac{2 \epsilon \tau \lambda}{z^{2}} .
$$

These effective fields are dipolar in nature rather then the usual $1 / r$ term for a charged line. However, they are not exponentially screened. (Close to the rod (distances $<\lambda$ ) the electrostatic potential is not screened and therefore is logarithmic as is the case for a bare charged rod.)

\subsection{Interaction between two neighboring rods}

In order to calculate the interaction energy between two charged rods adsorbed on a salty surface we use Eq. 6 for the free energy of the system with the charge distribution of two rods separated by a distance $D$. We make use of the fact that the $\mathrm{DH}$ equation (Eq. $\mathrm{B}$ ) is linear so that we can solve for each rod separately and then superimpose the potentials and charge distributions of the combined system of both rods. In order to compare with the sim- ulation results which will be described in the following chapter, we have to modify the problem slightly so that the rods are at least slightly raised above the surface and thus do not interfere with the charge distribution around each other. (This is a requirement of the simulated system in order to allow for ions to move from one side of the rod to the other.) For a rod raised by a small (compared with $\lambda$ ) distance $d$ above the surface, the amplitudes of the modes in $\psi$ are now modified to be $\alpha_{q}(d)=-\frac{\tau e^{-d q}}{\epsilon(2 q \epsilon \lambda+1)}$. For rods that are close to each other $(D<\lambda)$ we know that the fields will lead to a logarithmic inter-rod interaction. However, the fields farther away are more complicated and the resulting interaction is not obvious.

The interaction can be calculated numerically as a function of distance between the two rods, however, when the rods are separated by a distance $D \gg \lambda$ the potential (Eq. 13) and charge distribution, $\sigma$ (Eq. 14), can be analytically approximated yielding a simple form for the interaction energy as a function of the distance $D$. Once this approximation is made it can be shown that $\phi(x, z=0) \sim 1 / x^{2}$ and $\sigma(x) \sim 1 / x^{2}$ and the integrals in Eq. 6 are simplified to yield the interaction per unit length of the rods as a function of distance:

$$
G(D \gg \lambda) \simeq \frac{8(\tau \lambda)^{2}}{\epsilon D^{2}}\left(1+\frac{3}{4} \frac{d}{\lambda}+\frac{1}{4}\left(\frac{d}{\lambda}\right)^{2}\right) .
$$

Here $D$ is the inter-rod distance and $d$ is the distance between the rods and the charged surface (Fig. 1).

The interaction is similar to that of two rods of dipoles at a distance $D$ apart. However, we can not say that the charge distribution is actually dipolar since the field in the perpendicular direction $\left(E_{z}\right)$ behaves differently. 
In general one usually expects the interaction between charged objects in solution to be dominated by the osmotic pressure of the solute, in this case the two-dimensional salt solution. However, in this two-dimensional case the imperfectly screened electrostatic interactions dominate over the osmotic pressure which decays more quickly as a function of $D\left(\Pi_{\text {osmotic }} \simeq k_{B} T\left(\sigma_{+}(D / 2)+\sigma_{-}(D / 2)\right) \simeq\right.$ $O\left(\phi^{2}\right) \simeq O\left(D^{-4}\right)$. Differentiating equation 16 leads to the correct two-dimensional pressure between the rods which is dominated by electrostatic contributions:

$$
\Pi=-\frac{\delta G}{\delta D}=\frac{16(\tau \lambda)^{2}}{\epsilon D^{3}}\left(1+\frac{3}{4} \frac{d}{\lambda}+\frac{1}{4}\left(\frac{d}{\lambda}\right)^{2}\right)
$$

\section{Numerical Simulations}

In order to verify the predictions of the above theory, we have performed numerical Brownian dynamics simulations of the effective interactions between charged stiff (infinitely long) rods (along the $y$ direction) above (in the $z$ direction) a surface, $(x, y, z=0)$, in which charged monovalent particles can move (see Fig. 1). The simulations are performed at a temperature, $T=300 K$, and with uniform dielectric constant, $\epsilon=80$, simulating the continuum properties of bulk water. Simulating a non-zero salt concentration in the surface, we have applied periodic boundary conditions in the plane $(x, y)$, with a periodicity of $\left(L_{x}, L_{y}\right)=(400,40)$, where length is normalized to $r_{0}=1 \AA$. The normalized long range interactions between rods in this partially periodic system are thus given by
[16],

$U_{r r}(x, z)=-\frac{\tau_{1} \tau_{2}}{\epsilon} \ln \left(2 \cosh \left(2 \pi \frac{d}{L_{x}}\right)-2 \cos \left(2 \pi \frac{D}{L_{x}}\right)\right)$,

and the normalized interaction, $U_{c r}(x, z)$, between a point charge and a rod is given by replacing $\tau_{2}$ with $q / L_{y}$ in the above expression, $q$ being the fractional charge of the point charge. Energy is here normalized to $E_{0}=e^{2} / 4 \pi \epsilon_{0}$. The corresponding interaction energy between point particles in partially periodic media can be found in Ref. [17]. We further employ a short range repulsive interaction potential (in units of $\mathrm{kcal} / \mathrm{mol}$ ) between ions in the plane:

$$
U_{L J}(r)= \begin{cases}4 \varepsilon\left(\frac{\rho}{r}\right)^{6}\left[\left(\frac{\rho}{r}\right)^{6}-1\right]+\varepsilon, & r<2^{1 / 6} \\ 0 & , \text { otherwise }\end{cases}
$$

with $\rho=4$ and $\varepsilon=0.01$.

Figure 2 shows the simulation results for two rods of charge density, $\tau=e / 2 \AA$, at a distance $d=10 \AA$ above the surface, and with a distance $D$ between them. The simulations have been performed by initiating the positions of the in-plane ions randomly and allowing them to equilibrate for $>10^{5}$ time steps $(d t=0.005$ in normalized time units) before averaging the mean forces over $>10^{6}$ steps. We show the attractive mean forces between the rods and the surface (Fig. 2a) as well as the repulsive mean force between the rods (Fig. 2b) for several different ionic strengths of the surface. The prevailing trend is that the mean force between the rods decays as $D^{-3}$ for large $D$ and that the mean force between the rods and the surface approaches the asymptotic (single rod limit) as $D^{-2}$ for large $D$. This is in agreement with the predictions given by Eq. 16 and 17. It should be noted that 
given the periodic boundary conditions necessary for simulating a non-zero concentration of salt in the surface, we do not have complete freedom to consider the limit $D \rightarrow \infty$. The largest possible $D$ is given by $L_{x} / 2$ (where all forces between the rods, in the $x$ direction, are zero by symmetry) and one should therefore only consider simulation results for $D$ somewhat less than $L_{x} / 2$ in order to obtain meaningful results to be compared to the theory, which does not consider a periodic array of particles and rods. The results show some signs of insufficient averaging for large $D$. Clearly, this is due to the very small mean forces that we try to evaluate, combined with the rather small number of simulated particles from which the averages are generated. The small systems are necessitated by the long range interactions which require all charged objects to interact with all other charged objects, thereby increasing the simulation time by the square of the number of charged objects simulated. Since the averaging only gets linearly better with the number of particles, we are limited to small systems if we want to perform calculations of the electrostatics carefully. Even so, the simulations do overall agree with the predictions. In fact, the two main predictions, namely that the inter-rod force and the rod-surface force asymptotically behave as $D^{-3}$ and $D^{-2}$, respectively, seem to be very robust results even though they were derived in the thermodynamic limit of a large number of overall salt ions in the plane. The three different cases shown in figure 2 represent ( $\mathrm{o}$ ) only counter-ions to the rods; $(\times)$ only salt at concentration $1 / 400 \AA^{-2}$ (charge neutrality is satisfied by evenly smearing the rod counter- charge on the surface); and $(\triangle) \circ$ and $\times$ combined. Even the case where only counter-ions are present shows reasonable agreement between simulations and theory. We have also verified that good comparisons between simulations and theory hold for other values of $d$, the distance between rods and surface. The general trend is that as $d$ increases, so does the distance, $D$, at which the mean forces approach the predicted slopes shown in figure 2 .

\section{Conclusions}

We have presented both theoretical and simulation results for the interactions between charged DNA-like rods near a salty surface. We have focused on the polarization of the surface charge distribution and how it affects the fields and resulting interaction between two such rods. Our main conclusion is that when the mobile charges are confined, as is the case in our treatment, to a two-dimensional surface, they do not exponentially screen the fields, and hence the effective interaction between the rods is not finite-ranged. Both theory and simulations show consistent power law interactions both for the force between the rods and for the force they apply to the charged surface.

Despite the fact that the limits of applicability of the theory and simulation are not the same: the theory is valid at the limit of a large number of ions, and assumes an infinite surface with just two rods, while the simulation is restricted, for the reasons mentioned in section 1 , to a small number of particles and periodic boundary conditions, we still find a finite region of inter-rod distances where the two agree fairly well. This agreement indicates 
that although the theory is approximate it is robust non the less.

The systems we treat in this paper may seem relatively artificial because the charges are confined to the surface and there is no, or very little, residual bulk salt in the surrounding water solution. In addition, we do not take into account the effects of the non-zero thickness of lipid membranes and the relatively low (compared to the surrounding water) dielectric constant of the lipid. However, despite these limitations, we can apply these results to some experimentally investigated systems. Specifically, in recent x-ray experiments[3] that studied DNA-Cationic lipid complexes the structures that were found were formed by layers of membranes intercalated by ordered DNA domains. Most of these experiments were performed in very low bulk salt concentration, and moreover, because the counter-ions were trapped between the membranes they were effectively restricted to two-dimensional space. (The lower dimensionality of the space is "measured" relative to the fixed charged objects that polarize the surrounding solute. In this case we are studying the screening of the DNA and therefore the space available to the ions for redistributing themselves is effectively two-dimensional compared with the DNA, despite the fact that the ions are almost point like in comparison with the $20 \AA$ diameter of the DNA.) This experimental limit may also be valid in biological layered structures such as the Golgi apparatus. Although the effects of dielectric discontinuities in these electrostatic systems can be nontrivial (we treat these elsewhere [13]) they do not change the main results
Fig. 1. Schematic of the model system of membrane and DNA strands

Fig. 2. Mean forces between rods and surface (a) and between the two rods (b) as a function of the inter-rod distance, $D$. System parameters are: $L_{x}=400, L_{y}=40, \tau=e / 1.7 \AA, d=$ $10, T=300 K$. Distances are in units of $\AA$. Forces in figure (a) are relative to the force at $D=L_{x} / 2$. Results are shown for three different two dimensional salt concentrations (see figure).

for distances $D \gg \lambda$, and we do not expect the power law forces to change in this regime.

We are grateful to Cyrus Safinya, Ilya Koltover and Gerard Wang for discussing with us their experimental results. We thank Helmut Schiessel, Dov Levine and Nily Dan for useful discussions. PP and RM acknowledge the partial support of the MRL Program of the National Science Foundation under Award No. DMR99-72246 in addition to Awards 8-442490-21587 and 8-442490-21825. NGJ acknowledges support by the Director, Office of Advanced Scientific Computing Research, Division of Mathematical, Information, and Computational Sciences of the U.S. Department of Energy under contract number DE-AC03$76 \mathrm{SF} 00098$.

\section{References}

1. W. Firshein, Annu. Rev. Microbiol. 43, (1989) 89.

2. J.P. Behr, Bioconjugate Chem., 5 (1994) 382; D. Moradpour, J.I. Schauer, V.R., Jr., Zurawski, J.R. Wands and R.H. Boutin, Biochem. \& Biophys. Res. Commun., 221 (1996) 82; I.M. Verma and N. Somia, Nature, 389 (1997) 239. 
3. J. Rädler, I. Koltover, T. Salditt and C.R. Safinya, Science, 275 (1997) 810; T. Salditt, I. Koltover, J. Rädler and C.R. Safinya, Phys. Rev. Lett., 79 (1997) 2582.

4. R. Bruinsma and J. Mashl, Europhys. Lett., 41 (1998) 165;

R. Bruinsma, Euro. Phys. J.B, 4 (1998), 75.

5. H. Schiessel, Euro. Phys. J. B, 6 (1998) 373.

6. S.P.A. Fodor, Science, 277 (1997) 393.

7. B.V. Derjaguin, N.V. Churaev and V.M. Muller Surface Forces (Consultants Bureau, New York and London 1987), Section 6.9 and references therein.

8. H. Totsuji, J. Phys. Soc. Japan, 40, 857 (1976).

9. E.S. Velazquez and L. Blum, Physica A, 244 (1997) 453.

10. R. Menes, P.Pincus, R. Pittman and N. Dan, Europhys. Lett., 44 (1998) 393.

11. P.A. Pincus and S.A. Safran Europhys. Lett., 42 (1998) 103.

12. E.W. Kaler, A.K. Murthy , B.E. Rodriguez and J.A.N. Zasadzinski, Science, 245 (1989) 1371.

13. R. Menes and P.A. Pincus, preprint.

14. L.D. Landau and E.M. Lifshitz, Statistical Physics, 3rd edition, revised and enlarged by E.M. Lifshitz and L.P. Pitaevskii (Pergamon, New York 1980) p. 240.

15. S.A. Safran Statistical Thermodynamics of Surfaces, Interfaces, and Membranes (Addison-Wesley Publishing Company, 1994), Section 5.5.

16. R.J. Mashl, N. Grønbech-Jensen, M.R. Fitzsimmons, M. Lütt and D.Q. Li, J. Chem. Phys., 110 (1999) 2219 and references within.

17. N. Grønbech-Jensen, G. Hummer and K.M. Beardmore, Mol. Phys., 92 (1997) 941. 


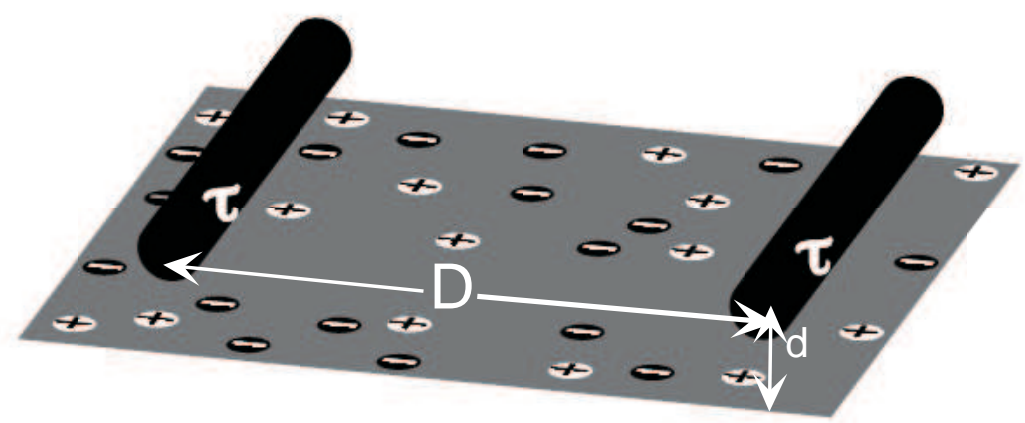




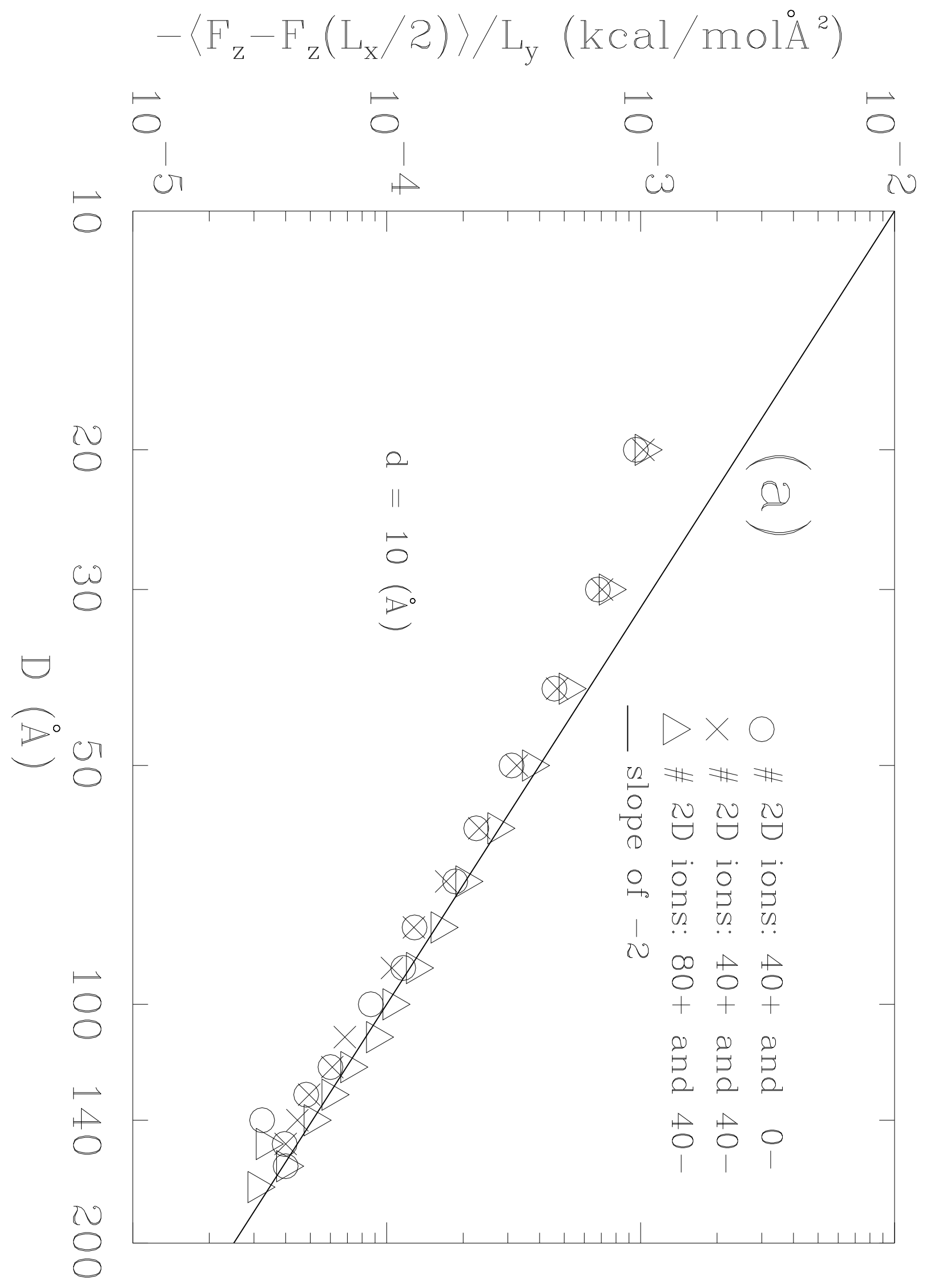




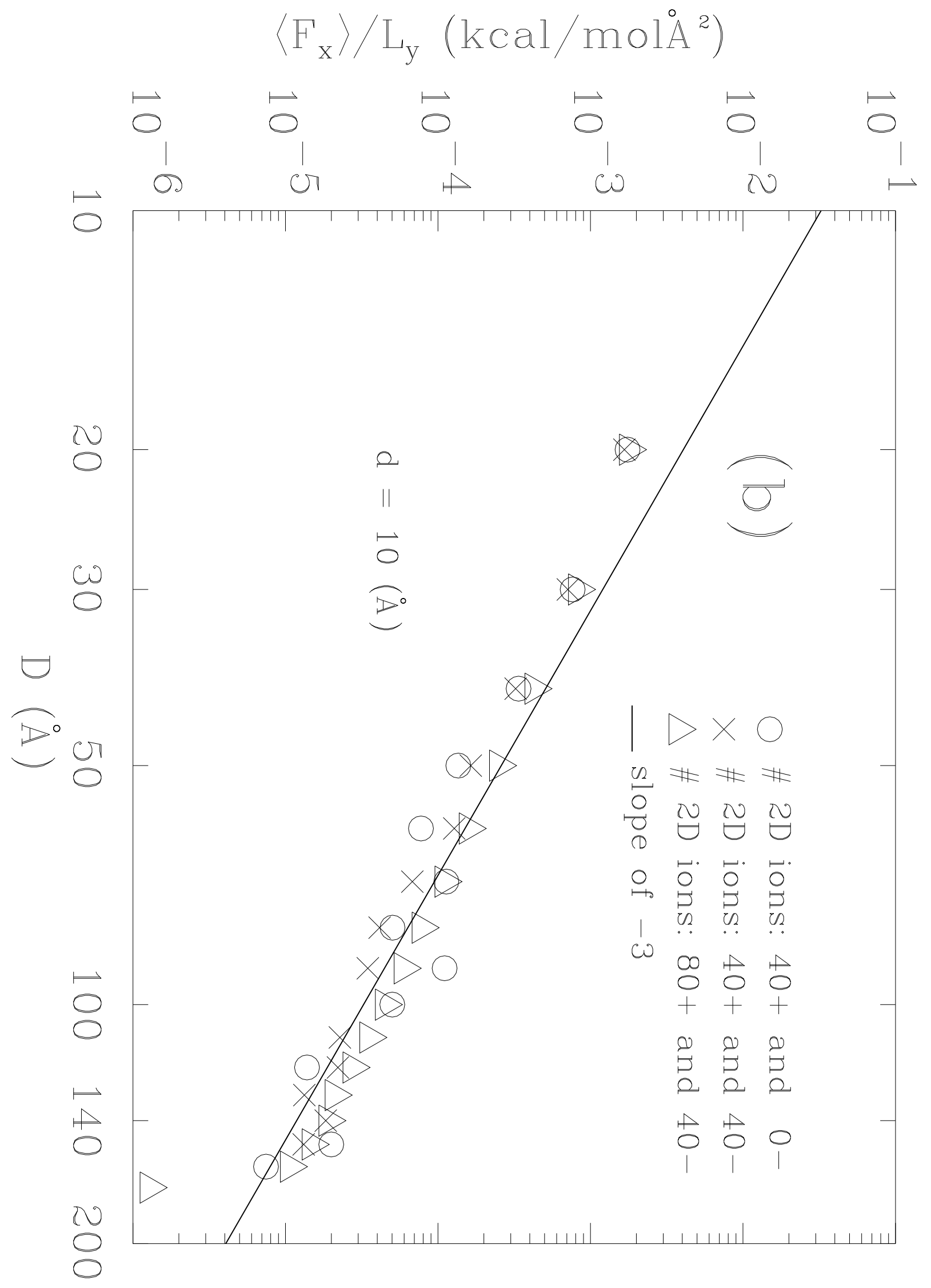

